\title{
Special issue on social impact of AI: killer robots or friendly fridges
}

\author{
Greg Michaelson $\cdot$ Ruth Aylett
}

Published online: 11 October 2011

(C) Springer-Verlag London Limited 2011

At the 2009 Artificial Intelligence and Simulation of Behaviour Convention, held in Edinburgh in April 2009, we chaired a vibrant Symposium on the "Social Impact of AI". The intentionally provoking subtitle of "Killer Robots or Friendly Fridges" highlighted the contradictory strands in public attitudes to AI beyond academic research circles. These are fed variously by: almost 100 years of speculative fiction and film; journalistic hyping of sober scientific and engineering advances; along with the slow and prosaic deployment of AI technologies in everyday artefacts.

During the Symposium, over thirty people attended seven challenging presentations by international practitioners, engendering lively debate and controversy. Subsequently, we invited the presenters to refine and expand their papers, and attendees to submit new relevant work, for this special issue of AI \& Society. After refereeing, from ten submissions, we are now pleased to introduce five:

- Claude Draude. Intermediaries: reflections on virtual humans, gender, and the Uncanny Valley

- Patricia A. Vargas, Ylva Fernaeus, Mei Yii Lim, Sibylle Enz, Wan Ching Ho, Mattias Jacobsson and Ruth Aylett. Advocating an ethical memory model for artificial companions from a human-centred perspective

- Aleksandra Kupferberg, Stefan Glasauer, Markus Huber, Markus Rickert, Alois Knoll and Thomas Brandt. Biological movement increases acceptance of humanoid robots as human partners in motor interaction

G. Michaelson $(\bowtie) \cdot$ R. Aylett

School of Maths and Computer Science, Montbatten Building, Heriot-Watt University, Edinburgh E14 4AS, UK

e-mail: G.Michaelson@hw.ac.uk

R. Aylett

e-mail: r.s.aylett@hw.ac.uk
- Ugo Pagallo. Killers, Fridges, and Slaves: A Legal Journey in Robotics

- Pablo Lucas. Usefulness of Simulating Social Phenomena: Evidence

Draude reconstructs the Uncanny Valley, drawing strongly on the legacy of Hoffman's Sandman story, where human emotional involvement overcomes the "natural" revulsion to the "artificial". In contrast, Vargas et al. explore how a characteristic "robo-ethics" could be elaborated to mitigate this long-standing dichotomy. In a complementary paper, Kupferberg et al. consider how the perceived naturalness of artificial constructs is affected by their movement and gait. Pagallo discusses how legal notions of agency might change as the autonomy of artificial systems develops. Finally, Lucas surveys the usefulness of AI-based social models to concrete social and policy analyses.

Overall, these papers offer an engaging snapshot of current research foci on the interactions between Artificial Intelligence and wider society.

We would like to thank our referees:

- Prof Alan Bundy, University of Edinburgh

- Prof Bob Colomb, University of Technology, Malaysia

- Dr Rudi Lutz, University of Sussex

- Prof Margit Pohl, Vienna University of Technology

for their perceptive and constructive reflections on the submissions.

\section{Guest editors}

Prof Ruth Aylett: ruth@macs.hw.ac.uk and Prof Greg Michaelson; G.Michaelson@hw.ac.uk

Editors note

The following individual papers, included in this volume, reflect the inter-disciplinary focus of AI \& Society, 
ranging from uploading minds onto computers, technology, culture and vigilance, brain-computer interface, Organisational governance and innovation.

Neil Levy: Searle's Wager

C. T. A. Schmidt: Technology and culture and possibly vigilance too

Gerd Grübler: Beyond the responsibility gap. Discussion note on responsibility and liability in the use of braincomputer interfaces
Pradip Kumar Biswas: Networks of small enterprises, architecture of governance and incentive alignment: some cases from India

R. Varman and M. Chakrabarti: Notes from small industry clusters: Making sense of knowledge and barriers to innovation 